\title{
Dimorphism-associated Variations in the Lipid Composition of Candida albicans
}

\author{
By M. A. GHANNOUM, G. JANINI, L. KHAMiS and S. S. RADWAN* \\ Department of Botany and Microbiology and Department of Chemistry, Kuwait University, \\ PO Box 5969, Kuwait
}

(Received 3 January 1986; revised 20 March 1986)

\begin{abstract}
Yeast and mycelial forms of Candida albicans ATCC 10231, growing together in $12 \mathrm{~h}$ and in $96 \mathrm{~h}$ cultures, were separated and their lipids were extracted and characterized. The total lipid content of the yeast forms was always lower than that of the mycelial forms. In $12 \mathrm{~h}$ cultures the lipids from the two morphological forms consisted mainly of polar compounds, viz. phospholipids and glycolipids. In $96 \mathrm{~h}$ cultures both the yeast and mycelial forms accumulated substantial amounts of apolar compounds, mainly steryl esters and triacylglycerols. The mycelial forms were more active than the yeast forms in this respect. Major differences in the lipid composition between the two morphological forms involved the contents of sterols and complex lipids that contain sterols. As a rule, the yeast lipids contained much larger proportions of free sterols than the mycelial lipids. However, the mycelial lipids contained several times more sterols than the yeast forms but bound as steryl glycosides, esterified steryl glycosides and steryl esters. Steryl glycosides and esterified steryl glycosides occurred in yeast lipids only in traces, if at all. The major steryl glycoside in the mycelial forms was unequivocally identified as cholesteryl mannoside. At both phases of growth the apolar and polar lipid fractions from the mycelial forms contained higher levels of polyunsaturated fatty acids $(18: 2$ and 18:3) but lower levels of oleic acid $(18: 1)$ than the corresponding fractions from the yeast forms. The lipid content and composition of $12 \mathrm{~h}$ and $96 \mathrm{~h}$ yeast and mycelial forms of $C$. albicans KCCC 14172, a clinical isolate, were almost identical with those of C. albicans ATCC 10231.
\end{abstract}

\section{INTRODUCTION}

In the last ten years the lipids of Candida albicans have been the subject of a considerable number of investigations. Many studies have been devoted to the relation of lipids to antibiotic resistance (Fryberg et al., 1975; Yamaguchi, 1977; Koh et al., 1977; Subden et al., 1977; Singh et al., 1979a; Mehta et al., 1984); others have been concerned with changes in lipids during ageing (Combs et al., 1980), starvation and germ-tube formation (Sundaram et al., 1981) and with the role of sterols in amino acid transport (Singh et al., 1979b). The lipids of isolated cellular fractions such as the cell wall (Bianchi, 1967) and the plasma membrane (Marriott, 1975; Yamaguchi, 1977) have also been investigated.

The comparison of lipids and their constituent fatty acids in yeast and mycelial forms of $C$. albicans is particularly important because only one of the two morphological forms is pathogenic (Balish \& Phillips, 1966; Rebora et al., 1973). Two studies have dealt with the lipids of cellular fractions only (Bianchi, 1967; Marriott, 1975) and another two only with the phospholipids of whole yeast cells and mycelia (Ballmann \& Chaffin, 1979; Yano et al., 1982). In all these studies the two morphological forms analysed were grown in separate cultures; hence, any differences recorded in the lipids of the two forms can, at least in part, be attributed to variations in culture conditions.

The main objective of the present work was to characterize and compare the lipids and their constituent fatty acids of yeast and mycelial forms of $C$. albicans grown in the same cultures. 


\section{METHODS}

Organisms and growth conditions. Candida albicans ATCC 10231 and C. albicans KCCC 14172, a clinical isolate obtained from the oral cavity of a cancer patient undergoing therapy at Kuwait Cancer Control Centre, were the strains used. Stock cultures were maintained on slopes of modified Sabouraud agar medium (Difco) stored at $4^{\circ} \mathrm{C}$ and subcultured routinely. The organisms were grown at $37^{\circ} \mathrm{C}$ by static incubation in SSV medium (Marriott, 1975) for $96 \mathrm{~h}$. Several samples were taken, at intervals, for the determination of total dry weight and the relative proportions of yeast and mycelial forms.

Separation of yeast and mycelial forms. The culture containing yeast and mycelial forms was centrifuged; the pellet was washed three times with $0.9 \% \mathrm{NaCl}$ solution and was then mixed with $0.9 \% \mathrm{NaCl}$ solution to form a relatively heavy suspension that was passed through two successive filters (Retsch) with pore sizes of $38 \mu \mathrm{m}$ and $20 \mu \mathrm{m}$, respectively. The mycelial residue on the filters was repeatedly rinsed with $0.9 \% \mathrm{NaCl}$ solution until it became free of yeast-like forms on microscopic examination. Yeast-like cells in the filtrate were harvested by centrifugation.

Scanning electron microscopy (SEM). Samples of the yeast and mycelial forms as well as of the mixed cultures were prepared for SEM (Ghannoum \& Al-Khars, 1984). The cells were suspended in glutaraldehyde (1.5\%, w/v) for $2 \mathrm{~min}$, collected by centrifugation and resuspended in a fresh sample of the same fixative for $16 \mathrm{~h}$ at $4^{\circ} \mathrm{C}$. The samples were washed with distilled water, placed on a coverslip, left to dry and coated ready for examination in a stereoscan electron microscope (Novascan 30).

Lipid extraction and analysis. Cultures of each strain grown for $12 \mathrm{~h}$ and $96 \mathrm{~h}$ were studied. Total lipids were extracted from samples of yeast and mycelial forms as well as from the mixed culture with chloroform $/ \mathrm{methanol}$ $(2: 1, \mathrm{v} / \mathrm{v})$ and purified using established procedures (Folch et al., 1957). Apolar lipid fractions were resolved by chromatography on thin layers of silicic acid using hexane/diethyl ether/acetic acid $(90: 10: 1$, by vol.) as a developing solvent (Mangold \& Malins, 1960). Polar lipid fractions were resolved by two-dimensional TLC using the solvent systems chloroform/methanol/7 $\mathrm{M}$-ammonium hydroxide $(65: 30: 4$, by vol.) in the first direction and chloroform/methanol/acetic acid/water (1 70:25:25:4, by vol.) in the second direction (Nichols, 1964).

The various lipid fractions were detected by exposure to iodine vapour or by charring at $220^{\circ} \mathrm{C}$ after spraying the plates with $50 \%(\mathrm{v} / \mathrm{v}) \mathrm{H}_{2} \mathrm{SO}_{4}$. The fractions were identified by comparing their chromatographic behaviour with that of authentic samples and by the colour reactions they gave with specific spray reagents (Stahl, 1962; Dittmer \& Lester, 1964; Siakotos \& Rouser, 1965). For structural studies of polar lipid fractions, samples (about $10 \mathrm{mg}$ ) of pure compounds, separated by preparative TLC and eluted from the adsorbent with chloroform/diethyl ether/ethanol (1:1:1, by vol.) (Kates, 1972), were subjected to acid hydrolysis (Gaver \& Sweeley, 1965). Hydrolysis products soluble in chloroform were identified by TLC, GLC and mass spectrometry (Finnigan MAT 311A; double-focusing reverse near-Johnson geometry). Lipid fractions resolved by TLC and subjected to charring were quantified densitometrically using a Beckman R-112 densitometer. Sterols were also determined quantitatively by GLC using $\beta$-sitosterol as an internal standard. Hydrolysis products soluble in water and found by paper chromatography (Menzies \& Seakins, 1976) to be hexoses were quantitated spectrophotometrically (Yamamoto \& Rouser, 1970).

Samples of apolar and polar lipid fractions separated by preparative TLC were subjected to methanolysis (Chalvardjian, 1964). The resulting methyl esters were purified by TLC and analysed by GLC using a Pye Unicam model 204 gas chromatograph fitted with a glass column $(1.83 \mathrm{~m} \times 4 \mathrm{~mm}$ i.d. $)$ packed with $15 \%$ DEGS on Anakrom D, 100-120 mesh. Sterols were analysed by GLC using a glass column $(1.83 \mathrm{~m} \times 2 \mathrm{~mm}$ i.d. $)$ packed with $3 \%$ OV-17 on Chromosorb W-HP, 100-120 mesh.

\section{RESULTS}

The separation of yeast and mycelial forms by filtration led to highly pure preparations of each form (Fig. 1).

The growth curves of the two C. albicans strains and, for strain ATCC 10231, changes in the proportions of the two morphological forms during the incubation period are shown in Fig. 2. It is apparent that the $12 \mathrm{~h}$ samples taken for lipid analysis were in the exponential phase of growth whereas the $96 \mathrm{~h}$ cultures were in the late stationary phase. The proportions by weight of the two morphological forms showed slight fluctuations during growth. Apparently some mycelial forms were producing blastospores (e.g. after $18 \mathrm{~h}$ ) and some blastospores were germinating again into mycelia (e.g. after $96 \mathrm{~h}$ ) without any significant change in the total biomass. However, as the results in Fig. 2 indicate, these fluctuations involved only relatively small proportions of the two morphological forms. Therefore, the samples collected for lipid analysis may be considered satisfactorily homogeneous, as far as their ages are concerned. 

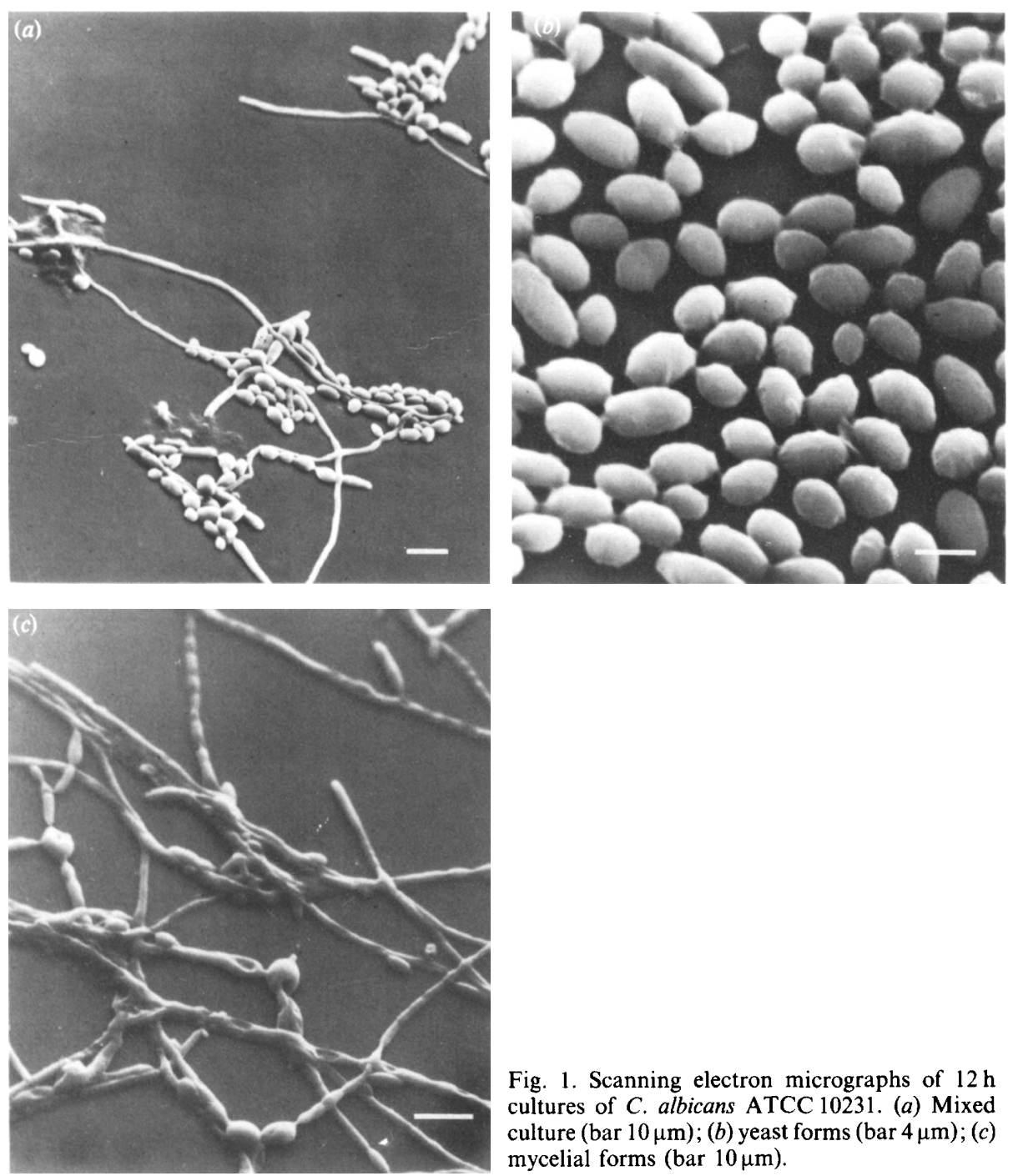

Fig. 1. Scanning electron micrographs of $12 \mathrm{~h}$ cultures of $C$. albicans ATCC 10231. (a) Mixed culture (bar $10 \mu \mathrm{m}$ ); (b) yeast forms (bar $4 \mu \mathrm{m}) ;(c)$ mycelial forms (bar $10 \mu \mathrm{m}$ ).

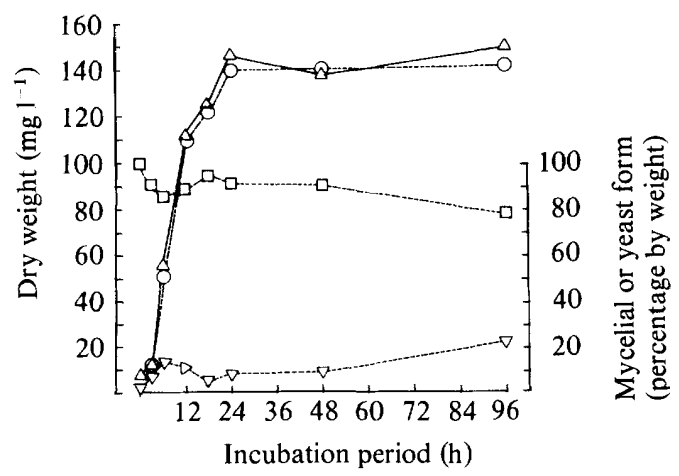

Fig. 2. Growth curves of $C$. albicans. $\triangle$, Strain ATCC 10231 ; $\bigcirc$, strain KCCC 14172. $\square$, percentage of yeast forms; $\nabla$, percentage of mycelial forms (strain ATCC 10231). 


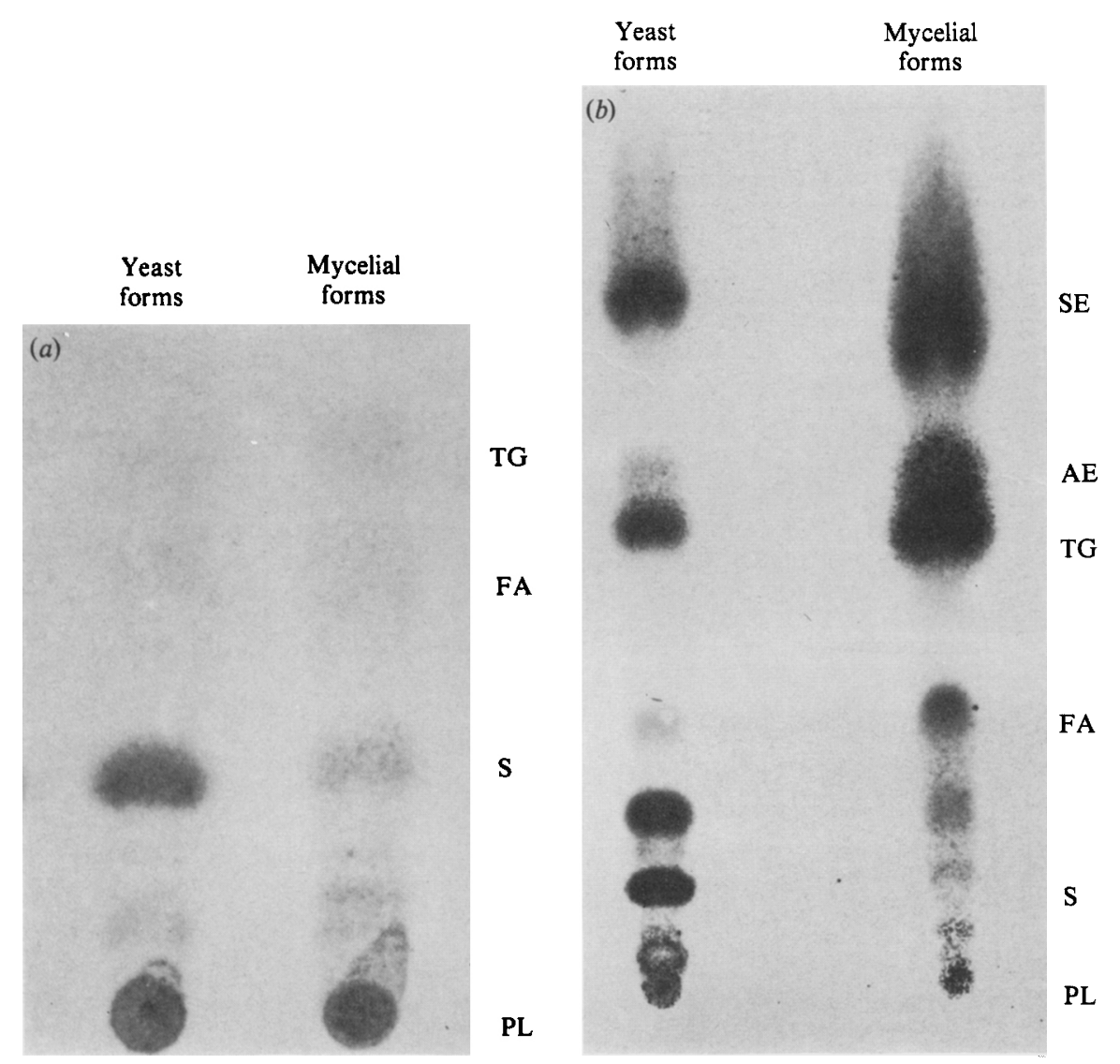

Fig. 3. Typical TLC plates showing apolar lipids of yeast and mycelial forms of $12 \mathrm{~h}(a)$ and $96 \mathrm{~h}(b)$ cultures of $C$. albicans ATCC 10231. The sorbent was Silica Gel G; the solvent was hexane/diethyl ether/acetic acid ( $90: 10: 1$, by vol.). The lipids were visualized by exposure to iodine vapour. SE, steryl esters; AE, alkyl esters; TG, triacylglycerols; FA, fatty acids; S, sterols; PL, polar lipids.

We did not find strain-associated variation in the total lipid content nor in the lipid composition of $C$. albicans. The TLC plates of lipids from both strains were always identical. Therefore, we concentrated on the lipids of $C$. albicans ATCC 10231 only.

The total lipid content of $12 \mathrm{~h}$ yeast and mycelial forms was $0.4 \%$ and $1.3 \%$, respectively, on a dry weight basis. The total lipid content of $96 \mathrm{~h}$ cultures was $1.3 \%$ and $6.3 \%$ for yeast and mycelial forms, respectively.

The lipids from $12 \mathrm{~h}$ cultures of the two morphological forms consisted predominantly of polar compounds (Table 1). This situation changed in $96 \mathrm{~h}$ cultures, especially for mycelial lipids. However, the absolute content in various classes of polar lipids by no means decreased with ageing because the total lipid content of $96 \mathrm{~h}$ cultures of the two forms was several times higher than that of $12 \mathrm{~h}$ cultures. The apolar lipids from the yeast forms of $12 \mathrm{~h}$ cultures consisted predominantly of sterols, which were present at much lower concentrations in the mycelial forms of the same age (see also Fig. 3).

In $96 \mathrm{~h}$ cultures there were still more sterols in the yeast lipids than in the mycelial lipids. Yet, at this phase of growth, both morphological forms had accumulated considerable proportions of other apolar lipids, viz. steryl esters, alkyl esters, triacylglycerols and fatty acids. The mycelial forms accumulated considerably more of these lipid classes (about $60 \%$ of the total lipids) than did the yeast forms (about $40 \%$ ).

The polar lipids of $12 \mathrm{~h}$ cultures of the two morphological forms consisted mainly of 

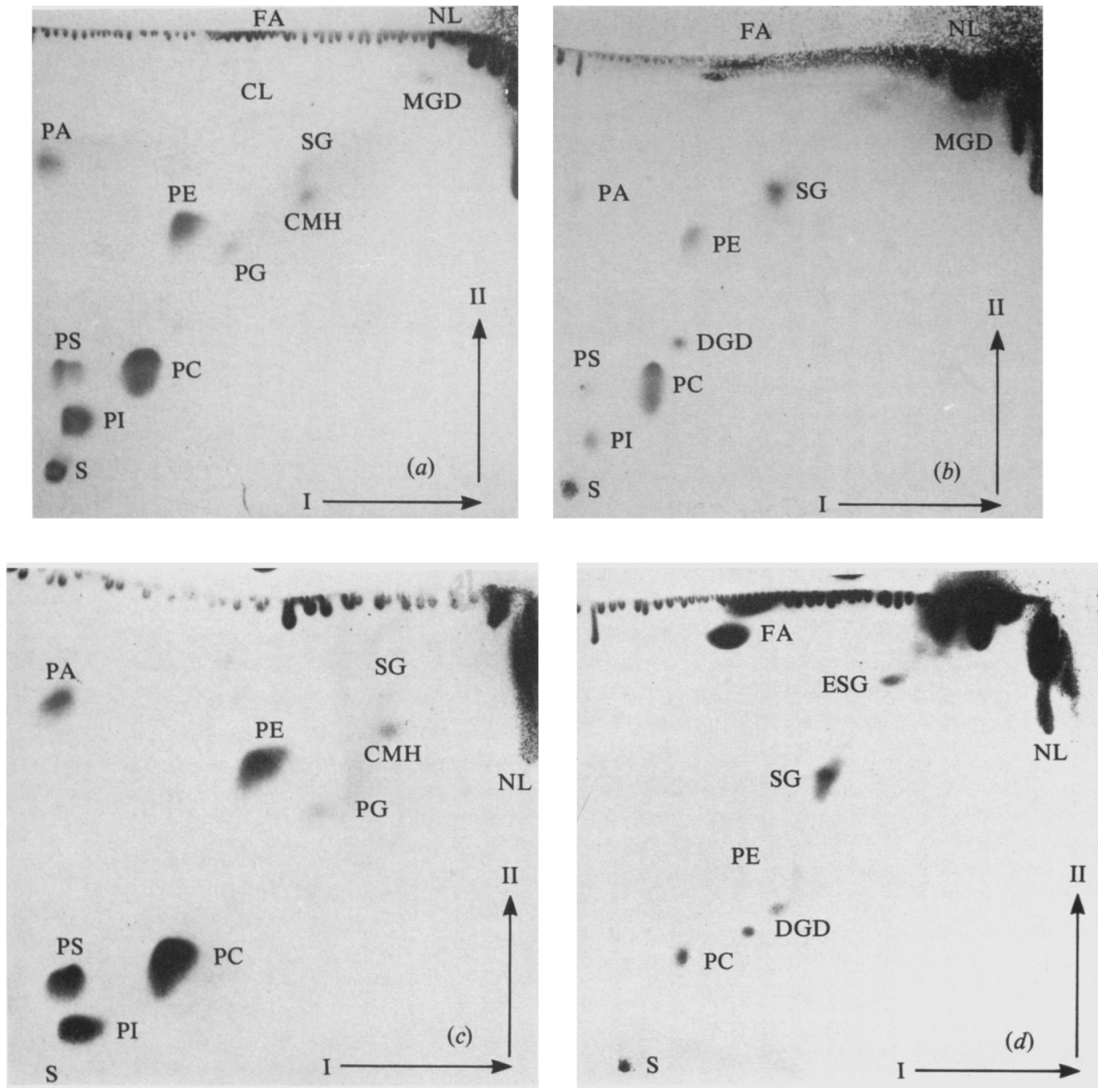

Fig. 4. Typical TLC plates of lipids from yeast $(a, c)$ and mycelial $(b, d)$ forms of $12 \mathrm{~h}(a, b)$ and $96 \mathrm{~h}$ $(c, d)$ cultures of $C$. albicans ATCC 10231. The sorbent was Silica Gel G; the solvents were (I) chloroform/methanol/7 M-ammonium hydroxide (65:30:4, by vol.) and (II) chloroform/methanol/ acetic acid/water (170:25:25:4, by vol.). The lipids were visualized by charring. S, start; PI, phosphatidylinositols; PS, phosphatidylserines; PA, phosphatidic acids; PC, phosphatidylcholines; PE, phosphatidylethanolamines; PG, phosphatidylglycerols; DGD, digalactosyldiacylglycerols; CMH, ceramide monohexosides; SG, steryl glycosides; ESG, esterified steryl glycosides; MGD, monogalactosyldiacylglycerols; CL, cardiolipins; FA, fatty acids; NL, neutral lipids.

phospholipids in addition to smaller proportions of glycolipids. In the $96 \mathrm{~h}$ cultures this remained true only for the yeast forms, whereas in the mycelial forms glycolipids predominated (see also Fig. 4).

The major phospholipids were phosphatidylcholines, phosphatidylethanolamines and phosphatidylinositols. Smaller proportions of phosphatidylserines and phosphatidylglycerols (mainly in the yeast forms) and phosphatidic acids were also found. The major glycolipids in the yeast lipids were ceramide monohexosides that occurred in the mycelial lipids only in traces. On the other hand, the mycelial lipids contained as major glycolipids steryl glycosides and, in $96 \mathrm{~h}$ cultures, esterified steryl glycosides too, in addition to substantial proportions of digalactosyl- 
Table 1. Comparison of lipids from yeast and mycelial forms of C. albicans ATCC 10231

Values are expressed as a percentage of the total lipids. Tr, traces; ND, not detected.

\section{Lipids}

Apolar compounds

Hydrocarbons

Steryl esters

Alkyl esters

Triacylglycerols

Fatty acids

Sterols

Polar compounds

Esterified steryl glycosides

Monogalactosyldiacylglycerols

Steryl glycosides

Ceramide monohexosides

Phosphatidylethanolamines

Phosphatidylglycerols

Phosphatidylcholines

Digalactosyldiacylglycerols

Phosphatidylserines

Phosphatidylinositols

Phosphatidic acids

$\overbrace{\begin{array}{c}\text { Yeast } \\ \text { forms }\end{array}}^{12 \mathrm{~h} \text { cultures }} \underbrace{}_{\begin{array}{c}\text { Mycelial } \\ \text { forms }\end{array}}$

\section{$\overbrace{\begin{array}{c}\text { Yeast } \\ \text { forms }\end{array}}^{96 \text { h cultures }} \underbrace{9 \text { cutcing }}_{\begin{array}{c}\text { Mycelial } \\ \text { forms }\end{array}}$}

Table 2. Identities and relative proportions $(\%)$ of sterols in total lipids ${ }^{*}$ from yeast and mycelial forms

Cultures were $12 \mathrm{~h}$ old.

\begin{tabular}{lcc}
\multicolumn{1}{c}{ Sterol } & $\begin{array}{c}\text { Yeast } \\
\text { forms }\end{array}$ & $\begin{array}{c}\text { Mycelial } \\
\text { forms }\end{array}$ \\
Degradation product of ergosterol & $4 \cdot 5$ & ND \\
Cholesterol & ND & $37 \cdot 8$ \\
Zymosterol & $17 \cdot 0$ & $13 \cdot 7$ \\
Ergosterol & $14 \cdot 6$ & $5 \cdot 6$ \\
24,28-Dehydroergosterol & $42 \cdot 7$ & $18 \cdot 8$ \\
3- $\beta$-Hydroxy-24-methyl cholesta-5,7-diene & $11 \cdot 7$ & $22 \cdot 2$ \\
4,4-Dimethylzymosterol & $9 \cdot 5$ & $2 \cdot 0$
\end{tabular}

ND, Not detected.

* Subjected to acid hydrolysis.

diacylglycerols and traces of monogalactosyldiacylglycerols. These glycolipids were only minor constituents of the yeast lipids.

Determinations based on spectrophotometric estimation of the sugar moiety (Yamamoto \& Rouser, 1970) as well as on GLC analysis of the sterol moiety confirmed the densitometric quantification, and showed that steryl glycosides made up 11-15\% of the total lipids from $12 \mathrm{~h}$ mycelial forms compared with only traces in lipids from $12 \mathrm{~h}$ yeast forms.

The fraction tentatively identified as steryl glycosides was isolated from mycelial lipids by preparative TLC and was subjected to acid hydrolysis. It produced a hexose and a sterol mixture in a molecular ratio of $1: 1$. In GLC analysis the sterol fraction showed a composition similar to that presented in Table 2. The sterol, whose retention time corresponded to that of cholesterol, was isolated by preparative GLC in a pure form (Fig. 5) and subjected to mass-spectrometric analysis. Low-resolution mass spectrometry of this compound was done at $125^{\circ} \mathrm{C}$, with direct insertion. From the MS traces the molecular ion was assumed to have an $m / e$ of about 386 . High resolution measurements gave values of $386 \cdot 297,386 \cdot 354$ (main peak) and $386 \cdot 390$. The 


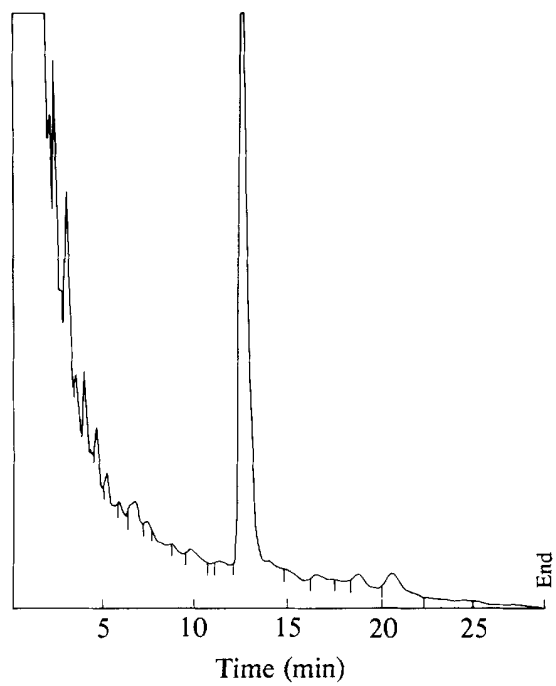

Fig. 5. Typical gas chromatogram showing the purity of cholesterol isolated from the sterol moiety of steryl glycosides by preparative GLC.

Table 3. Fatty acid composition of apolar and polar lipid fractions from yeast and mycelial forms

Apolar lipid fractions contained free fatty acids, triacyglycerols and steryl esters; polar lipid fractions contained phospholipids and glycolipids. Tr, traces.

\begin{tabular}{|c|c|c|c|c|c|c|c|c|}
\hline \multirow[b]{3}{*}{$\begin{array}{l}\text { Fatty } \\
\text { acid }\end{array}$} & \multicolumn{4}{|c|}{$12 \mathrm{~h}$ cultures } & \multicolumn{4}{|c|}{$96 \mathrm{~h}$ cultures } \\
\hline & \multicolumn{2}{|c|}{ Yeast forms } & \multicolumn{2}{|c|}{ Mycelial forms } & \multicolumn{2}{|c|}{ Yeast forms } & \multicolumn{2}{|c|}{ Mycelial forms } \\
\hline & $\begin{array}{c}\text { Apolar } \\
\text { lipids } \\
(\%)\end{array}$ & $\begin{array}{c}\text { Polar } \\
\text { lipids } \\
(\%)\end{array}$ & $\begin{array}{c}\text { Apolar } \\
\text { lipids } \\
(\%)\end{array}$ & $\begin{array}{c}\text { Polar } \\
\text { lipids } \\
(\%)\end{array}$ & $\begin{array}{c}\text { Apolar } \\
\text { lipids } \\
(\%)\end{array}$ & $\begin{array}{c}\text { Polar } \\
\text { lipids } \\
(\%)\end{array}$ & $\begin{array}{c}\text { Apolar } \\
\text { lipids } \\
(\%)\end{array}$ & $\begin{array}{c}\text { Polar } \\
\text { lipids } \\
(\%)\end{array}$ \\
\hline $\begin{array}{l}14: 0 \\
14: 1\end{array}$ & $\begin{array}{l}4.3 \\
1.8\end{array}$ & $\begin{array}{l}4 \cdot 2 \\
6 \cdot 2\end{array}$ & $\begin{array}{l}4 \cdot 2 \\
\operatorname{Tr}\end{array}$ & $\begin{array}{l}3 \cdot 6 \\
\operatorname{Tr}\end{array}$ & $\begin{array}{l}6.6 \\
5 \cdot 1\end{array}$ & $\begin{array}{l}7.0 \\
2.0\end{array}$ & $\begin{array}{l}6.6 \\
5.7\end{array}$ & $\begin{array}{l}6.5 \\
8.2\end{array}$ \\
\hline $16: 0$ & 11.8 & $13 \cdot 1$ & 14.5 & $15 \cdot 3$ & 28.5 & $25 \cdot 4$ & $28 \cdot 4$ & 20.7 \\
\hline $16: 1$ & 11.4 & 9.6 & $12 \cdot 2$ & $13 \cdot 2$ & $10 \cdot 4$ & $10 \cdot 0$ & 14.6 & $\mathrm{Tr}$ \\
\hline $16: 2$ & $\mathrm{Tr}$ & $\mathrm{Tr}$ & $\mathrm{Tr}$ & $\mathrm{Tr}$ & 8.7 & $3 \cdot 1$ & 6.0 & 9.9 \\
\hline 18:0 & 14.0 & $10 \cdot 3$ & $16 \cdot 7$ & $15 \cdot 2$ & $14 \cdot 5$ & $7 \cdot 4$ & $10 \cdot 4$ & $12 \cdot 1$ \\
\hline $18: 1$ & 37.6 & 41.5 & $26 \cdot 7$ & $26 \cdot 7$ & 14.7 & $22 \cdot 4$ & 15.9 & $8 \cdot 2$ \\
\hline $18: 2$ & 12.0 & $10 \cdot 7$ & $16 \cdot 3$ & $13 \cdot 5$ & $5 \cdot 5$ & $18 \cdot 8$ & 2.7 & $\operatorname{Tr}$ \\
\hline $18: 3$ & 7.5 & $4 \cdot 4$ & $9 \cdot 4$ & 12.5 & 5.7 & 3.9 & $9 \cdot 3$ & 33.7 \\
\hline
\end{tabular}

calculated mass of authentic cholesterol was 386.355. A low resolution run of authentic cholesterol on the mass spectrometer gave a fragmentation pattern comparable to that of the isolated compound.

The hexose moiety of the steryl glycoside fraction was identified by paper chromatographic analysis as mannose. The major steryl glycoside in the mycelial lipids was thus cholesteryl mannoside. The identities and relative proportions of individual sterols from total lipids that were subjected to acid hydrolysis are shown in Table 2 . The mycelial lipids contained the same sterols as the yeast lipids, and in addition relatively high proportions of cholesterol.

The fatty acid composition of apolar and polar lipid fractions from yeast and mycelial forms is shown in Table 3 . In $12 \mathrm{~h}$ cultures both fractions of mycelial lipids contained higher levels of polyunsaturated fatty acids (18:2 and 18:3) but lower levels of oleic acid (18:1) than the corresponding yeast fractions. These differences became even more pronounced in $96 \mathrm{~h}$ cultures, in which the mycelial forms contained up to $33.7 \%$ linolenic acid $(18: 3)$ in their polar lipid fraction. 


\section{DISCUSSION}

In earlier studies on the lipids of $C$. albicans the two morphological forms were grown in separate cultures at various temperatures (Bianchi, 1967; Ballmann \& Chaffin, 1979) and/or using media of different composition (Bianchi, 1967; Marriott, 1975; Ballmann \& Chaffin, 1979; Yano et al., 1982). Such factors dramatically alter the lipid composition of various organisms (for reviews see Radwan \& Mangold, 1976; Wassef, 1977). To eliminate this source of error we analysed the lipids from yeast and mycelial forms growing in the same cultures.

Our results show that mycelial lipids are poorer than yeast lipids in sterols, but are much richer in complex lipids that contain sterols such as steryl glycosides and esterified steryl glycosides. Otherwise, the two morphological forms have phospholipid patterns similar to those described previously (Ballmann \& Chaffin, 1979; Yano et al., 1982). Steryl glycosides and esterified steryl glycosides are widely distributed in the lipids of higher plants and plant cell cultures (e.g. Radwan et al., 1975) but reports of their occurrence in lipids of micro-organisms are very rare (Thompson et al., 1973). Hence, the unequivocal identification of this lipid appeared to be of particular importance. By combined TLC, GLC and MS the major steryl glycoside in mycelial lipids was identified as cholesteryl mannoside.

As yet, it is not known what biological role(s) the steryl glycosides may play in the living cell. However, being polar compounds with hydrophilic (hexose) and hydrophobic (sterol) ends it is expected that such compounds would occur mainly in the cell envelope. Additional studies are still needed to explain why mycelial lipids of $C$. albicans contain sterols mainly as steryl glycosides whereas yeast lipids contain these compounds mainly in the free form. Sterols are commonly believed to stabilize cell membranes and provide them with rigidity. The mycelial forms of $C$. albicans, being filamentous, must endure greater physical stress on their membranes than the yeast forms. The question that remains is do steryl glycosides confer more rigidity on membranes than free sterols? Mycoplasmas are prokaryotes that grow as naked thin filaments; they need to be supplied with cholesterol and accumulate this compound as a glycoside in their membranes (Rose, 1976).

Our result concerning the occurrence of higher levels of polyunsaturated fatty acids in mycelial lipids than in yeast lipids basically confirms the finding of an earlier study (Yano et al., 1982).

Thanks are due to Mr K. Abu Elteen and Mr S. Rajendra for valuable technical assistance. This work was supported by Kuwait University, Research Management Unit, Grants \# SO 024 and SDO 104.

\section{REFERENCES}

Balish, E. \& Phillips, A. W. (1966). Growth and virulence of Candida albicans after oral inoculation in the chick with a monoflora of either Escherichia coli or Streptococcus faecalis. Journal of Bacteriology 91 , 1744-1749.

Ballmann, G. E. \& Chaffin, W. L. (1979). Lipid synthesis during reinitiation of growth from stationary phase cultures of Candida albicans. Mycopathologia 67, 39-43.

BiANCHI, D. E. (1967). The lipid content of cell walls obtained from juvenile, yeast-like and filamentous cells of Candida albicans. Antonie Van Leeuwenhoek 33, 324-332.

Chalvardjian, A. M. (1964). Fatty acids of brown and yellow fat in rats. Biochemical Journal 90, 518-521.

Combs, T. J., Guarneri, J. J. \& Pisano, M. A. (1980). Lipid composition of Candida albicans as affected by culture age and medium aeration. Developments in Industrial Microbiology 21, 373-378.

Dittmer, J. C. \& Lester, R. L. (1964). A simple specific spray for the detection of phospholipids on thin-layer chromatograms. Journal of Lipid Research 5, 126-127.

Folch, J., Lees, M. \& Sloane Stanley, G. H. (1957). A simple method for the isolation and purification of total lipids from animal tissues. Journal of Biological Chemistry 226, 497-509.

Fryberg, M., Oehlschlager, A. C. \& Unrau, A. M. (1975). Sterol biosynthesis in antibiotic sensitive and resistant Candida. Archives of Biochemistry and Biophysics 173, 171-177.

Gaver, R. C. \& Sweeley, C. C. (1965). Methods for methanolysis of sphingolipids and direct determination of long chain bases by gas chromatography. Journal of the American Oil Chemists Society 42, 294.

Ghannoum, M. A. \& AL-Khars, A. (1984). Effect of antineoplastic agents on the growth and ultrastructure of Candida albicans. Mykosen 27, 452-464.

Kates, M. (1972). Techniques of Lipidology, p. 444. Amsterdam \& London: North-Holland.

Koh, T. Y., Marriott, M. S., Taylor, J. \& Gale, E. F. (1977). Growth characteristics and polyene sensitivity of a fatty acid auxotroph of Candida albicans. Journal of General Microbiology 102, 105110. 
Mangold, H. K. \& Malins, D. C. (1960). Fractionation of fats, oils and waxes on thin layers of silicic acid. Journal of the American Oil Chemists Society 37, 383-385.

MARRIOTT, M. S. (1975). Isolation and chemical characterization of plasma membranes from the yeast and mycelial forms of Candida albicans. Journal of General Microbiology 86, 115-132.

Mehta, R. J., Boyer, J. M. \& NASH, C. H. (1984). Aculeacin resistant mutants of Candida albicans: alterations in cellular lipids. Microbios Letters 27, 25 29.

Menzies, I. S. \& Seakins, J. T. (1976). Sugars. In Chromatographic and Electrophoretic Techniques, vol. 1, p. 183. Edited by I. Smith \& J. T. Seakins. London: William Heinemann.

Nichols, B. W. (1964). Separation of plant phospholipids and glycolipids. In New Biochemical Separations. Edited by A. T. James and I. J. Morris. London: Van Nostrand.

RadWan, S. S. \& Mangold, H. K. (1976). The lipids in plant tissue cultures. Advances in Lipid Research 14, 171-211.

Radwan, S. S., SPener, F., Mangold, H. K. \& Staba, E. J. (1975). Lipids in plant tissue cultures. IV. The characteristic patterns of lipid classes in callus cultures and suspension cultures. Chemistry and Physics of Lipids 14, 72-80.

Rebora, A., Marples, R. M. \& Kligman, A. M. (1973). Experimental infection with Candida albicans. Archives of Dermatology 108, 69-73.

Rose, A. H. (1976). Chemical Microbiology. An Introduction to Microbial Physiology, 3rd end, p. 52. London: Butterworths.

Siakotos, A. N. \& Rouser, G. (1965). Analytical separation of nonlipid water soluble substances and gangliosides from other lipids by dextran gel column chromatography. Journal of the American Oil Chemists Society 42, 913-919.
Singh, M., JAYAKumar, A. \& Prasad, R. (1979a). Lipid composition and polyene antibiotic sensitivity in isolates of Candida albicans. Microbios 24, 7-17.

Singh, M., JaYakumaR, A. \& Pradad, R. (1979 $b$ ). The effect of altered ergosterol content on the transport of various amino acids in Candida albicans. Biochimica et biophysica acta 555, 42-55.

STAHL, E. (1962). Dünnschicht-Chromatographie. Berlin: Springer.

Subden, R. E., SAFe, L., Morris, D. C., Brown, R. G. \& SAFE, S. (1977). Eburicol, lichesterol, ergosterol, and obtusifoliol from polyene antibiotic-resistant mutants of Candida albicans. Canadian Journal of Microbiology 23, 751-754.

Sundaram, S., Sullivan, P. A. \& Shepherd, M. G. (1981). Changes in lipid composition during starvation and germ-tube formation in Candida albicans. Experimental Mycology 5, 140-147.

Thompson, E. D., Knights, B. A. \& Parks, L. W. (1973). Identification and properties of a sterolbinding polysaccharide isolated from Saccharomyces cerevisiae. Biochimica et biophysica acta 304, 132.

WASSEF, M. K. (1977). Fungal Lipids. Advances in Lipid Research 15, 159-232.

YamaguchI, H. (1977). Antagonistic action of lipid components of membranes from Candida albicans and various other lipids on two imidazole antimycotics, clotrimazole and miconazole. Antimicrobial Agents and Chemotherapy 12, 16-25.

YAMAMOTo, A. \& RouSER, G. (1970). Spectrophotometric determination of molar amounts of glycosphingolipids and ceramide by hydrolysis and reaction with trinitrobenzene sulphonic acid. Lipids 5, 442.

Yano, K., Yamada, T., Banno, Y., Sekiya, T. \& Nozawa, Y. (1982). Modification of lipid composition in a dimorphic fungus, Candida albicans during yeast cell to hypha transformation. Japanese Journal of Medical Mycology 23, 159-165. 